Article

\title{
Improving Wind Farm Dispatchability Using Model Predictive Control for Optimal Operation of Grid-Scale Energy Storage
}

\author{
Douglas Halamay, Michael Antonishen, Kelcey Lajoie, Arne Bostrom and Ted K. A. Brekken* \\ School of Electrical Engineering and Computer Science (EECS), Oregon State University, \\ Corvallis, OR 97331, USA; E-Mails: douglasahalamay@eaton.com (D.H.); \\ michael.antonishen@gmail.com (M.A.); kalajoie@bpa.gov (K.L.); apbostrom@gmail.com (A.B.) \\ * Author to whom correspondence should be addressed; E-Mail: brekken@eecs.oregonstate.edu; \\ Tel.: +1-541-737-2995.
}

Received: 19 June 2014; in revised form: 21 August 2014 / Accepted: 21 August 2014 /

Published: 5 September 2014

\begin{abstract}
This paper demonstrates the use of model-based predictive control for energy storage systems to improve the dispatchability of wind power plants. Large-scale wind penetration increases the variability of power flow on the grid, thus increasing reserve requirements. Large energy storage systems collocated with wind farms can improve dispatchability of the wind plant by storing energy during generation over-the-schedule and sourcing energy during generation under-the-schedule, essentially providing on-site reserves. Model predictive control (MPC) provides a natural framework for this application. By utilizing an accurate energy storage system model, control actions can be planned in the context of system power and state-of-charge limitations. MPC also enables the inclusion of predicted wind farm performance over a near-term horizon that allows control actions to be planned in anticipation of fast changes, such as wind ramps. This paper demonstrates that model-based predictive control can improve system performance compared with a standard non-predictive, non-model-based control approach. It is also demonstrated that secondary objectives, such as reducing the rate of change of the wind plant output (i.e., ramps), can be considered and successfully implemented within the MPC framework. Specifically, it is shown that scheduling error can be reduced by $81 \%$, reserve requirements can be improved by up to $37 \%$, and the number of ramp events can be reduced by $74 \%$.
\end{abstract}

Keywords: energy storage; model predictive control (MPC); reserve generation; wind generation; wind ramps 


\section{Introduction}

The installed wind energy capacity is growing rapidly throughout the world. The natural variability of wind increases reserve requirements, thus limiting grid penetration. In the Pacific Northwest, the installed wind capacity has been on the rise nearly exponentially since 1998, as shown in Figure 1 [1].

Figure 1. Cumulative nameplate rating of the installed wind capacity in the US Pacific Northwest from 1998 to 2012 [1].

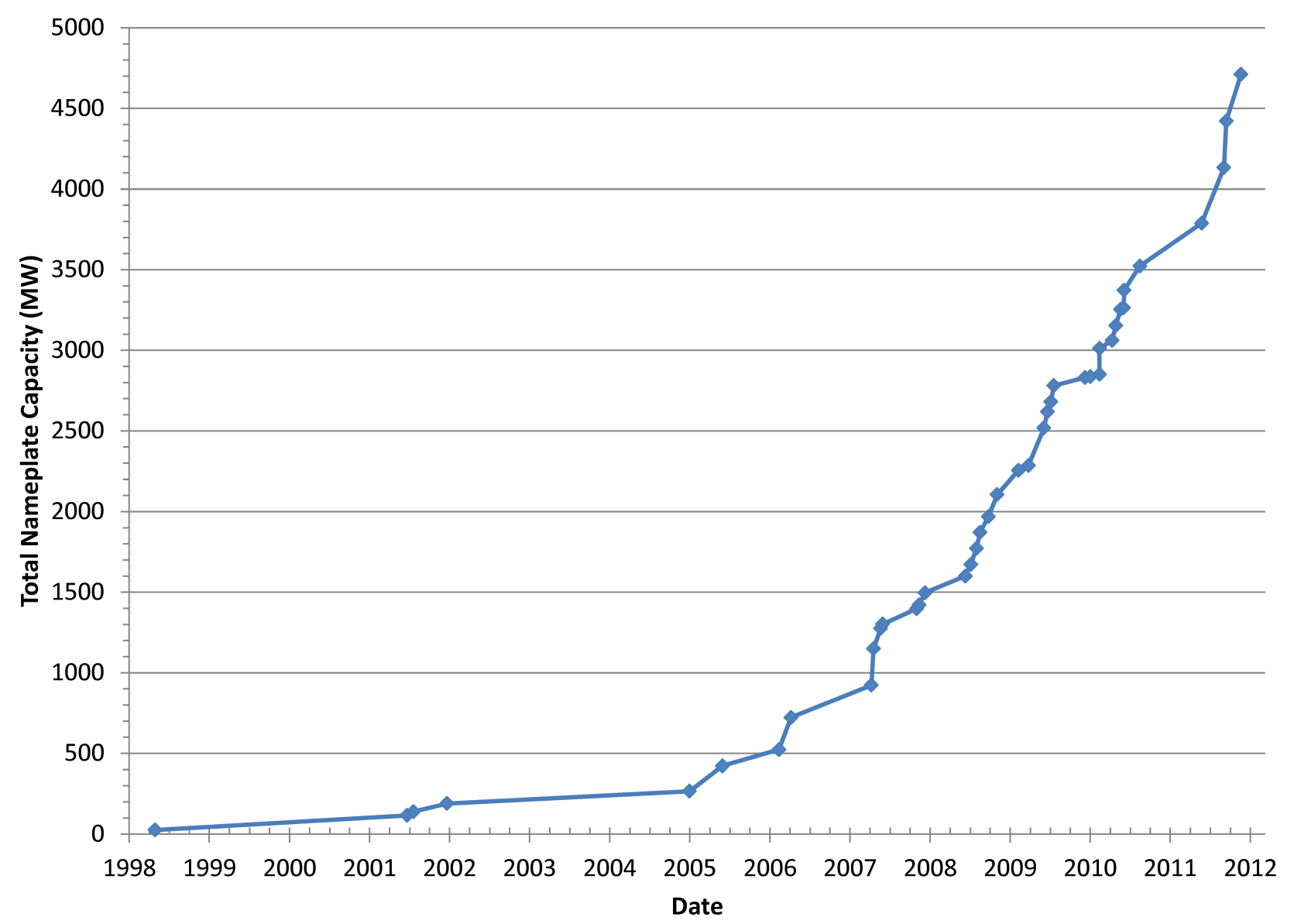

In the Pacific Northwest, utility reserve services are provided by generation from the Federal Columbia River Power System (FCRPS) (i.e., hydropower). The FCRPS is subject to a wide variety of constraints, including fish migration, flood control, recreation, river traffic control, and electrical power generation. The increase in variability from the growing wind power resource has begun to exhaust the reserve capacity of the system [2]. According to the Northwest Power and Conservation Council (NPCC), hydropower makes up 56\% of online generating capacity in the Pacific Northwest as of February 2012, with wind accounting for $12 \%$ [3]. Most of the installed wind in the Pacific Northwest is in the Columbia River Gorge, a relatively small geographic area [4]. This means that when the wind rapidly increases, power generation from wind can go from nearly $0 \mathrm{MW}$ to over $4000 \mathrm{MW}$ in a matter of hours, meaning that several thousand megawatts of generation must be reduced somewhere else on the system in a short period of time. This reserve service is provided primarily by hydropower generation. The research presented in this paper investigates large-scale on-site energy storage as an alternative source of reserve capacity, as shown in Figure 2. 
Figure 2. On-site energy storage to provide reserve generation, such that the combined wind farm and energy storage output (i.e., $P_{\text {plant }}$ ) matches the scheduled wind farm output.

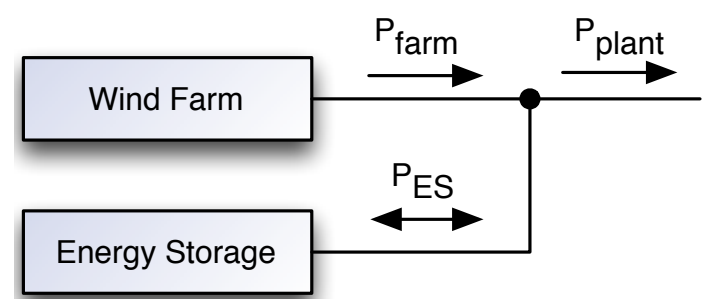

This paper will focus on simulations that combine a wind farm with a grid-scale energy storage source in the form of a zinc-bromine battery system. The addition of the energy storage source is meant to increase the dispatchability and reliability of the wind farm. A novel model predictive control (MPC) system has been developed for use with this combined system. Previous work has shown that energy storage can be used to increase wind power predictability and reduce variability in renewable energy plants [5-10]. In particular, Khatamianfar et al. [6] demonstrated an improvement in wind farm revenue of $3 \%$ when energy storage was added to the wind plant, and Teleke et al. [10] demonstrated that reducing the prediction horizon from $30 \mathrm{~min}$ to $15 \mathrm{~min}$ improved the performance of a wind and energy storage system by $14 \%$. Additionally, many other control approaches have been tested and the results suggest that the combination of energy storage and wind power is technically and financially feasible [5,11-14].

There are few publications in the literature on the formulation and qualitative analysis of the performance of energy storage for wind farms, particularly utilizing MPC. Explorations of the effectiveness of the addition of energy storage with and without predictive control and different types of power scheduling and forecasting will be discussed.

\section{Methodology}

\subsection{Proposed Control Structure}

In this research, a MPC structure has been implemented. For MPC, current and future control actions are determined in the context of predicted plant behavior. At each sample time, the plant states are measured (or estimated) and any known plant disturbances are predicted over some length of time into the future (i.e., the prediction horizon). A time series of control actions are then determined to drive the plant to a desired trajectory over the prediction horizon. The first control action of the time series is applied to the plant and the process is repeated at the next sample time [15].

One of the benefits of having a predictive controller, as opposed to a reactive controller [16], is that the system can take preparatory actions to mitigate the negative effects of events such as wind ramps before they happen. Additionally, the MPC structure contains limit and cost information on both the plant and control actions.

Figure 3 shows a block diagram of the model predictive controller including inputs, system control, and outputs. The controller takes the predicted wind data as inputs and produces an energy storage power command as output. Additional parameters include system limits and costs associated with control commands. The inputs and outputs for this research will be discussed further below. 
Figure 3. Model predictive control (MPC) controller organization.

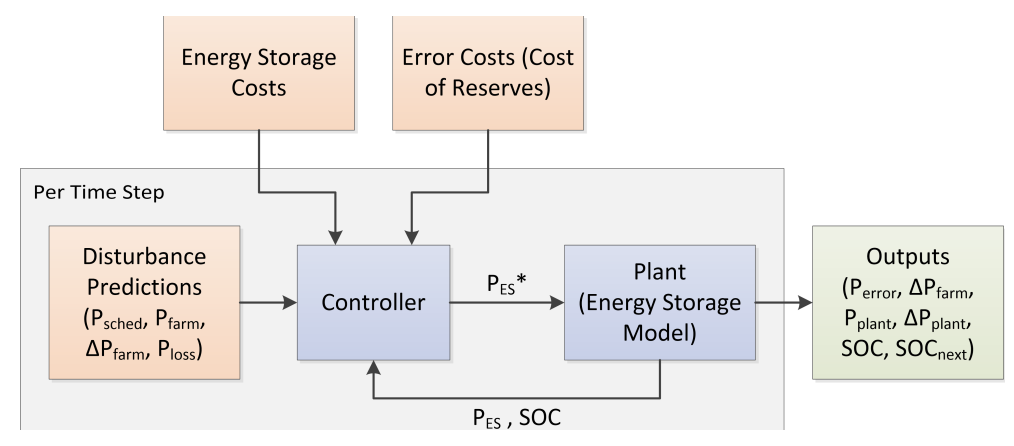

\subsection{Model Predictive Control Formulation}

In this section, we formulate the discrete-time plant predictive model. In discrete time, the state update equation can be seen in Equation (1) and the output equation in Equation (2):

$$
\begin{gathered}
\mathbf{x}(k+1)=\mathbf{A x}(k)+\mathbf{B}_{u} \mathbf{u}(k)+\mathbf{B}_{v} \mathbf{v}(k) \\
\mathbf{y}(k)=\mathbf{C x}(k)+\mathbf{D}_{u} \mathbf{u}(k)+\mathbf{D}_{v} \mathbf{v}(k)
\end{gathered}
$$

In Equation (1), $\mathbf{x}(k+1)$ gives the system state one step in the future, $\mathbf{A}$ is the state matrix, $\mathbf{x}(k)$ is the current system state, $\mathbf{B}_{u}$ is the input control matrix, $\mathbf{u}(k)$ is the input control vector, $\mathbf{B}_{v}$ is the input disturbance matrix, and $\mathbf{v}(k)$ is the input disturbance vector. In Equation (2), $\mathbf{y}(k)$ is the output vector, $\mathbf{C}$ is the output matrix, $\mathbf{D}_{u}$ is the feedthrough input control matrix, and $\mathbf{D}_{v}$ is the feedthrough input disturbance matrix. To form the predictive model over the prediction horizon $H_{p}$, Equations (1) and (2) are incremented one sample into the future and recursively back-substituted, yielding the predictive model shown in Equations (3) and (4) [15]:

$$
\begin{aligned}
& \underbrace{\left[\begin{array}{c}
\mathbf{y}(k) \\
\mathbf{y}(k+1) \\
\mathbf{y}(k+2) \\
\vdots \\
\mathbf{y}\left(k+H_{p}\right)
\end{array}\right]}_{\overrightarrow{\mathbf{y}}(k)}=\underbrace{\left[\begin{array}{c}
\mathbf{C} \\
\mathbf{C A} \\
\mathbf{C A}^{2} \\
\vdots \\
\mathbf{C A}^{H_{p}}
\end{array}\right]}_{\mathbf{S}_{x}} \mathbf{x}(k)+\underbrace{\left[\begin{array}{ccccc}
\mathbf{D}_{u} & 0 & 0 & \cdots & 0 \\
\mathbf{C B}_{u} & \mathbf{D}_{u} & 0 & \cdots & 0 \\
\mathbf{C A B}_{u} & \mathbf{C B}_{u} & \mathbf{D}_{u} & \cdots & 0 \\
\vdots & \vdots & \vdots & \ddots & \vdots \\
\mathbf{C A}^{H_{p}-1} \mathbf{B}_{u} & \mathbf{C A}^{H_{p}-2} \mathbf{B}_{u} & \cdots & \mathbf{C B}_{u} & \mathbf{D}_{u}
\end{array}\right]}_{\mathbf{S}_{u}} \underbrace{\left[\begin{array}{c}
\mathbf{u}(k) \\
\mathbf{u}(k+1) \\
\mathbf{u}(k+2) \\
\vdots \\
\mathbf{u}\left(k+H_{p}\right)
\end{array}\right]}_{\overrightarrow{\mathbf{u}}(k)} \\
& +\underbrace{\left[\begin{array}{ccccc}
\mathbf{D}_{v} & 0 & 0 & \cdots & 0 \\
\mathbf{C B}_{v} & \mathbf{D}_{v} & 0 & \cdots & 0 \\
\mathbf{C A B}_{v} & \mathbf{C B}_{v} & \mathbf{D}_{v} & \cdots & 0 \\
\vdots & \vdots & \vdots & \ddots & \vdots \\
\mathbf{C A}^{H_{p}-1} \mathbf{B}_{v} & \mathbf{C A}^{H_{p}-2} \mathbf{B}_{v} & \cdots & \mathbf{C B}_{v} & \mathbf{D}_{v}
\end{array}\right]}_{\mathbf{S}_{v}} \underbrace{\left[\begin{array}{c}
\mathbf{v}(k) \\
\mathbf{v}(k+1) \\
\mathbf{v}(k+2) \\
\vdots \\
\mathbf{v}\left(k+H_{p}\right)
\end{array}\right]}_{\overrightarrow{\mathbf{v}}(k)} \\
& \overrightarrow{\mathbf{y}}(k)=\mathbf{S}_{x} \mathbf{x}(k)+\mathbf{S}_{u} \overrightarrow{\mathbf{u}}(k)+\mathbf{S}_{v} \overrightarrow{\mathbf{v}}(k)
\end{aligned}
$$


Note that $\overrightarrow{\mathbf{y}}(k)$ is the concatenated current and predicted output vector, $\overrightarrow{\mathbf{u}}(k)$ is the control vector, and $\overrightarrow{\mathbf{v}}(k)$ is the concatenated current and predicted disturbance vector. The dimensions are:

$$
\begin{aligned}
\overrightarrow{\mathbf{y}} & \in \Re^{n_{y} \cdot\left(H_{p}+1\right) \times 1} \\
\overrightarrow{\mathbf{u}} & \in \Re^{n_{u} \cdot\left(H_{p}+1\right) \times 1} \\
\overrightarrow{\mathbf{v}} & \in \Re^{n_{v} \cdot\left(H_{p}+1\right) \times 1} \\
\mathbf{S}_{x} & \in \Re^{n_{y} \cdot\left(H_{p}+1\right) \times n_{x}} \\
\mathbf{S}_{u} & \in \Re^{n_{y} \cdot\left(H_{p}+1\right) \times n_{u} \cdot\left(H_{p}+1\right)} \\
\mathbf{S}_{v} & \in \Re^{n_{y} \cdot\left(H_{p}+1\right) \times n_{v} \cdot\left(H_{p}+1\right)}
\end{aligned}
$$

where $n_{x}$ is the number of states; $n_{y}$ is the number of outputs; $n_{u}$ is the number of inputs; $n_{v}$ is the number of disturbances; and $H_{p}$ is the prediction horizon.

The quadratic tracking objective function is shown in Equation (6), where $\overrightarrow{\mathbf{t}}(k)$ is the desired plant trajectory and is of the same dimension as $\overrightarrow{\mathbf{y}}(k)$ :

$$
J(k)=(\overrightarrow{\mathbf{t}}(k)-\overrightarrow{\mathbf{y}}(k))^{T} \mathbf{Q}(\overrightarrow{\mathbf{t}}(k)-\overrightarrow{\mathbf{y}}(k))+\overrightarrow{\mathbf{u}}(k)^{T} \mathbf{R} \overrightarrow{\mathbf{u}}(k)
$$

The matrices $\mathbf{Q}$ and $\mathbf{R}$ determine the costs associated with tracking errors and control actions, respectively. The dimensions are:

$$
\begin{aligned}
& \mathbf{Q} \in \Re^{n_{y} \cdot\left(H_{p}+1\right) \times n_{y} \cdot\left(H_{p}+1\right)} \\
& \mathbf{R} \in \Re^{n_{u} \cdot\left(H_{p}+1\right) \times n_{u} \cdot\left(H_{p}+1\right)}
\end{aligned}
$$

Next, we manipulate Equation (6) such that $\overrightarrow{\mathbf{u}}(k)$ is the only independent variable. A new variable $\overrightarrow{\mathbf{e}}(k)$, the free evolution error, is defined in Equation (8). This is the resulting tracking error under the influence of only disturbance inputs and no control actions:

$$
\begin{aligned}
\overrightarrow{\mathbf{e}}(k) & =\overrightarrow{\mathbf{t}}(k)-\left(\overrightarrow{\mathbf{y}}(k)-\mathbf{S}_{u} \overrightarrow{\mathbf{u}}(k)\right) \\
& =\overrightarrow{\mathbf{t}}(k)-\left(\mathbf{S}_{x} \mathbf{x}(k)+\mathbf{S}_{v} \overrightarrow{\mathbf{v}}(k)\right)
\end{aligned}
$$

Substituting Equation (8) into Equation (6) yields:

$$
J(k)=\overrightarrow{\mathbf{u}}(k)^{T}\left(\mathbf{S}_{u}^{T} \mathbf{Q S} \mathbf{S}_{u}+\mathbf{R}\right) \overrightarrow{\mathbf{u}}(k)-2 \overrightarrow{\mathbf{e}}(k)^{T} \mathbf{Q S}_{u} \overrightarrow{\mathbf{u}}(k)+\overrightarrow{\mathbf{e}}(k)^{T} \mathbf{Q} \overrightarrow{\mathbf{e}}(k)
$$

The bias terms are removed and the equation rescaled to yield the cost function for optimization:

$$
\hat{J}(k)=\hat{J}(\overrightarrow{\mathbf{u}}(k))=\frac{1}{2} \overrightarrow{\mathbf{u}}(k)^{T}\left(\mathbf{S}_{u}^{T} \mathbf{Q S}_{u}+\mathbf{R}\right) \overrightarrow{\mathbf{u}}(k)-\overrightarrow{\mathbf{e}}(k)^{T} \mathbf{Q S}_{u} \overrightarrow{\mathbf{u}}(k)
$$

Equation (10) represents a quadratic programming problem in which the vector $\overrightarrow{\mathbf{u}}(k)$ is determined to minimize $\hat{J}(k)$ in the context of constraints on $\overrightarrow{\mathbf{u}}(k)$.

For MPC implementation, the current control action $\mathbf{u}(k)$ is taken from the predicted control vector $\overrightarrow{\mathbf{u}}(k)$ and applied to the plant. At the next time step, Equation (8) is recalculated and the quadratic programming problem of Equation (10) is resolved, a new $\mathbf{u}(k)$ is determined, and the process repeats for each subsequent time sample. 


\subsection{Model Predictive Control Implementation}

In this application, a large-scale energy storage system is collocated with a wind farm such that the on-site energy storage provides reserves for the wind generation. The energy storage power output is controlled in such a way that the combined wind farm and energy storage output can match the scheduled wind power output. The plant model is given in Equations (11) and (12):

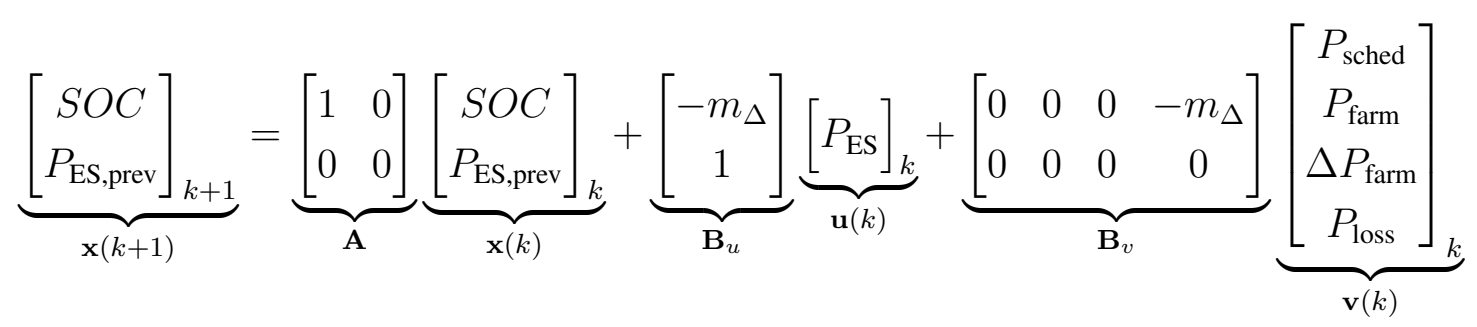

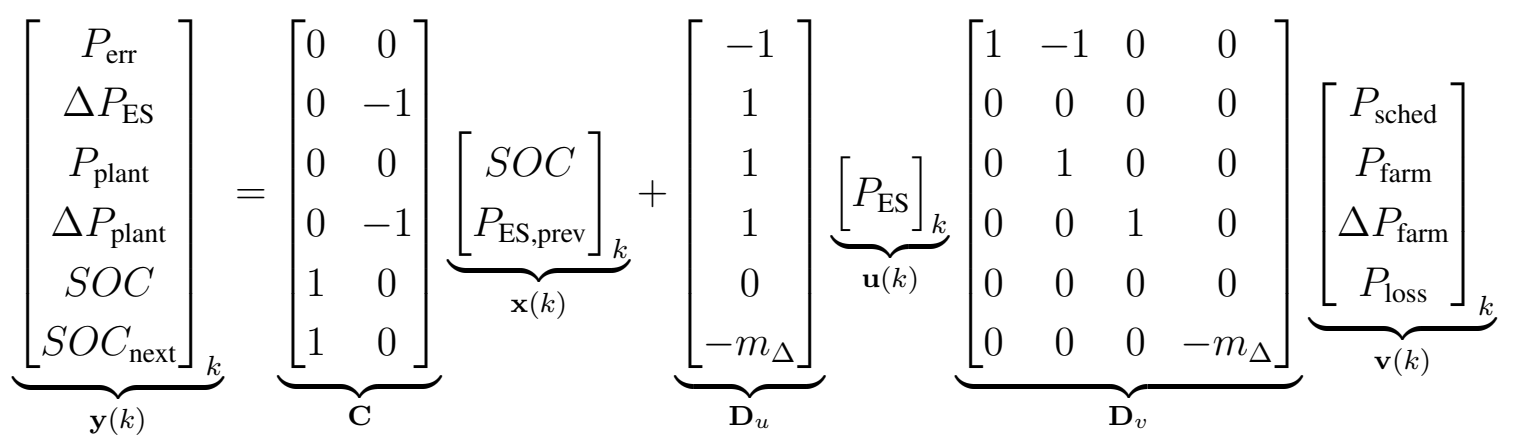

In Equation (11), $S O C$ is the state of charge of the energy storage source, $S O C_{\text {next }}$ is the next $S O C$ based on the losses and current energy storage command, $P_{\mathrm{ES} \text {,prev }}$ is the energy storage command from the last time step, $m_{\Delta}$ is the per-unit per-sample capacity constant of the energy storage source, $P_{\mathrm{ES}}$ is the current energy storage command, $P_{\text {sched }}$ is the scheduled output of the wind farm, $P_{\text {farm }}$ is the actual output of the wind farm, and $P_{\text {loss }}$ is the approximate loss in the energy storage source. In Equation (12), $P_{\text {err }}=P_{\text {sched }}-P_{\text {plant }}$, where $P_{\text {plant }}$ is the combined wind farm and energy storage output. The loss is included in the control model as an independent disturbance term to maintain linearity. It is positive for both positive and negative values of $P_{\mathrm{ES}}$, and for predictive purposes can be set as a constant to include both the self-discharge rate and an approximation of the power-dependent losses under normal usage.

In this research, the sample time of the discrete model shown above is $10 \mathrm{~min}$. The dynamic response of a zinc-bromine battery system is generally much faster than $10 \mathrm{~min}$ (it is expected to be in the order of seconds). Therefore, the dynamic response of the system is not included in the model in Equations (11) and (12). In other words, the commanded energy storage power output is assumed to always be equal to the actual power output.

The objective function weighting is shown in Equations (13)-(15). The individual terms in $\mathbf{q}$ are the corresponding weights for the entries of the output vector $\mathbf{y}(k)$. The dimension of $\mathbf{Q}$ is $n_{y} \cdot\left(H_{p}+1\right) \times n_{y} \cdot\left(H_{p}+1\right)$, and the dimension of $\mathbf{R}$ is $n_{u} \cdot\left(H_{p}+1\right) \times n_{u} \cdot\left(H_{p}+1\right)$. In this research, the matrices $\mathbf{Q}$ and $\mathbf{R}$ are purely diagonal as there is no need to weight cross-coupling terms or have the weights vary across the prediction horizon: 


$$
\begin{gathered}
\mathbf{q}=\left[\begin{array}{cccccc}
q_{P_{\mathrm{err}}} & 0 & 0 & 0 & 0 & 0 \\
0 & q_{\Delta P_{\mathrm{ES}}} & 0 & 0 & 0 & 0 \\
0 & 0 & q_{P_{\mathrm{plant}}} & 0 & 0 & 0 \\
0 & 0 & 0 & q_{\Delta P_{\text {plant }}} & 0 & 0 \\
0 & 0 & 0 & 0 & q_{S O C} & 0 \\
0 & 0 & 0 & 0 & 0 & q_{S O C_{\mathrm{next}}}
\end{array}\right] \\
\mathbf{Q}=\left[\begin{array}{ccc}
\mathbf{q}_{k} & \mathbf{0} & \mathbf{0} \\
\mathbf{0} & \ddots & \mathbf{0} \\
\mathbf{0} & \mathbf{0} & \mathbf{q}_{k+H_{p}}
\end{array}\right] \\
\mathbf{R}=\left[\begin{array}{ccc}
{\left[r_{P_{\mathrm{ES}}}\right]_{k}} & \mathbf{0} & \mathbf{0} \\
\mathbf{0} & \ddots & \mathbf{0} \\
\mathbf{0} & \mathbf{0} & {\left[r_{P_{\mathrm{ES}}}\right]_{k+H_{p}}}
\end{array}\right]
\end{gathered}
$$

Constraints for this implementation are shown in Equations (16) and (17):

$$
\begin{gathered}
{\left[\begin{array}{c}
P_{\mathrm{ES}} \\
-P_{\mathrm{ES}}
\end{array}\right] \leq\left[\begin{array}{c}
P_{\mathrm{ES}, \text { upper }} \\
-P_{\mathrm{ES}, \text { lower }}
\end{array}\right]} \\
{\left[\begin{array}{c}
S O C \\
-S O C \\
S O C_{\text {next }} \\
-S O C_{\text {next }}
\end{array}\right] \leq\left[\begin{array}{c}
S O C_{\text {upper }} \\
-S O C_{\text {lower }} \\
S O C_{\text {upper }} \\
-S O C_{\text {lower }}
\end{array}\right]}
\end{gathered}
$$

\section{Simulation Results}

\subsection{Simulation Setup}

For the simulations, a large-scale energy storage system is collocated with a wind farm such that the on-site energy storage provides reserves for the wind generation. The wind farm nameplate capacity is $100 \mathrm{MW}$ and the energy storage system is rated for $25 \mathrm{MW}$ (power) and $50 \mathrm{MW} \cdot \mathrm{h}$ (energy); this sizing, relative to the wind farm capacity, is based on considerations of existing large-scale energy storage systems, hardware availability, and sizing analysis from literature [17].

Wind farm power forecast and actual data were obtained from the Bonneville Power Administration (BPA) as matched historical data [18] (i.e., for every time index, the wind farm power is matched with forecasted wind farm power from that index onward). Simulations were conducted with data from the week of 26 March 2012 to 1 April 2012, comprised of 888 10-min samples. When the simulations were conducted, actual power data and forecasted wind power output for all wind on the BPA system were available as an aggregate, not for individual wind farms. The presented research assumes that the energy storage system to be simulated is for a single wind farm. Thus, the aggregate power and forecast data was scaled and used to represent a single farm. The aggregate data includes smoothing due to geographic diversity, which would not be present in data from an individual wind farm. Utilizing individual wind 
farm data will improve simulation accuracy when high-resolution data becomes available. The forecasted wind power was provided by a third-party meteorological forecasting firm (an example is presented in the Appendix). The wind farm scheduled output, $P_{\text {sched }}$, is determined by a persistence method. Both $60 \mathrm{~min}$ and $30 \mathrm{~min}$ scheduling intervals were used.

The primary control objective is to utilize the energy storage system to minimize $P_{\text {err }}$, which is the difference between the scheduled wind farm output $\left(P_{\text {sched }}\right)$ and the combined wind farm and energy storage output $\left(P_{\text {plant }}=P_{\text {farm }}+P_{\mathrm{ES}}\right)$. Plant ramp rates (i.e., $\left.\Delta P_{\text {plant }}\right)$ can also be considered within the control objective as well.

The predicted disturbance vector, $\overrightarrow{\mathbf{v}}(k)$, requires predictions of both the wind farm scheduled output $\left(P_{\text {sched }}(k \mid k), P_{\text {sched }}(k+1 \mid k), \cdots, P_{\text {sched }}\left(k+H_{p} \mid k\right)\right)$ and the wind farm actual output $\left(P_{\text {farm }}(k \mid k)\right.$, $\left.P_{\text {farm }}(k+1 \mid k), \cdots, P_{\text {farm }}\left(k+H_{p} \mid k\right)\right)$ over the prediction horizon, $H_{\mathrm{p}}$. Both of these predictions are based on the meteorological forecast at time $k$.

The cost $q_{P_{\text {err }}}$ in $\mathrm{Q}$, which is the cost of the mismatch between scheduled and actual plant output over the sample time, was determined from the BPA variable energy resource balancing service (VERBS) rate, which is currently charged to wind farms monthly based on installed capacity. The VERBS charge is currently $1.23 \$ / \mathrm{kW} / \mathrm{mo}$ of capacity and can be subdivided into regulating reserves for balancing moment to moment $(0.08 \$ / \mathrm{kW} / \mathrm{mo})$, following reserves for balancing larger mismatches within the hour $(0.37 \$ / \mathrm{kW} / \mathrm{mo})$, and imbalance reserves for balancing the difference between the generators schedule and the actual generation during $1 \mathrm{~h}(0.78$ \$/kW/mo) [19].

In order to translate VERBS into a real-time cost, scheduling error was summed over four months for a 100 MW wind farm in the Pacific Northwest. This error was then divided by the cost for four months of the VERBS service. The result is the constant $q_{P_{\mathrm{err}}}$ in the unit of $\$ / \mathrm{PU}^{2}$. Initially, this study explores the effectiveness of a model predictive controller to reduce error and/or ramp rate, and thus initially sets $r_{P_{\mathrm{ES}}}$ in $\mathbf{R}$ to zero, which is the cost of the energy storage output over the sample time. Future work will focus on including this cost in the analysis, which is explicitly one of the strengths of the MPC approach outlined here.

Losses were included in the model as a constant power loss and modeled after the chemical leakage losses of the zinc-bromine battery in the Wallace Energy Systems and Renewables Facility (WESRF) lab. Approximately $1 \%$ of the total energy capacity is lost each hour, except at SOC levels below $2 \%$ where losses become extremely low.

Controller constraints are specified for energy storage power and state of charge. The energy storage power constraints, $P_{\mathrm{ES} \text {,upper }}$ and $P_{\mathrm{ES} \text {,lower, }}$, are set to $0.25 \mathrm{PU}$ and $-0.25 \mathrm{PU}$, respectively, based on the limits of the zinc-bromine battery available in the WESRF lab. The state of charge constraints, $S O C_{\text {upper }}$ and $S O C_{\text {lower }}$, were set to $1 \mathrm{PU}$ and $0 \mathrm{PU}$ representing the safe full and empty states of charge, respectively. Simulations were performed with a $10 \mathrm{~min}$ sample time. The capacity of the energy storage system is $0.50 \mathrm{PUhr}$ and therefore the per-unit per-sample capacity constant in Equation (11) is $m_{\Delta}=\left(\begin{array}{ll}0.50 & P U h r\end{array}\right) \cdot\left(\begin{array}{ll}\frac{1}{6} & h r \\ \text { sample }\end{array}\right) /\left(\begin{array}{ll}0.25 & P U\end{array}\right)=1 / 3$. Each simulation covers a week.

In order to explore the effectiveness of MPC on minimizing $P_{\text {err }}$, five cases were examined. Figure 4(a) shows Case 1 with no energy storage or MPC. Figure 4(b)-4(d) shows the effects of enabling the use of energy storage, enabling predictive control, and shortening scheduling length from 60 min to 
30 min, respectively (Cases 2, 3, and 5). The figures demonstrate that error can be reduced by adding energy storage and even further by reducing scheduling length from $60 \mathrm{~min}$ to $30 \mathrm{~min}$.

Figure 4. Simulation results for: (a) Case 1 with energy storage inactive and $60 \mathrm{~min}$ scheduling $\left(P_{\text {plant }}=P_{\mathrm{ES}}+P_{\text {farm }}\right)$; (b) Case 2 with energy storage active, predictive control off, and 60 min scheduling $\left(P_{\text {plant }}=P_{\mathrm{ES}}+P_{\text {farm }}\right)$; the inclusion of energy storage reduces $P_{\text {err }}$; (c) Case 3 with energy storage active, predictive control on, and 60 min scheduling $\left(P_{\text {plant }}=P_{\mathrm{ES}}+P_{\text {farm }}\right)$; energy storage with predictive control further improves $P_{\text {err }}$; and (d) Case 5 with energy storage active, predictive control on, and 30 min scheduling $\left(P_{\text {plant }}=P_{\mathrm{ES}}+P_{\text {farm }}\right)$; reducing the scheduling interval improves $P_{\text {err }}$.

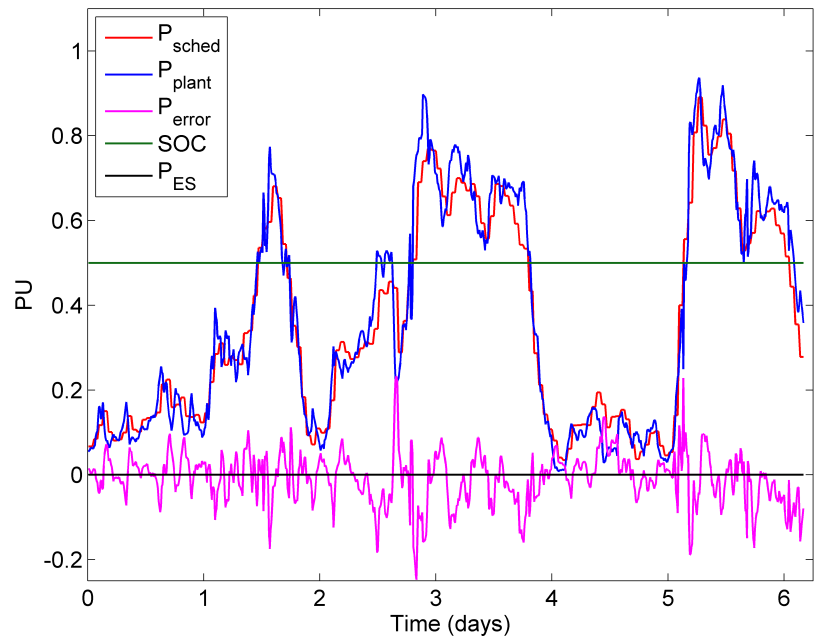

(a)

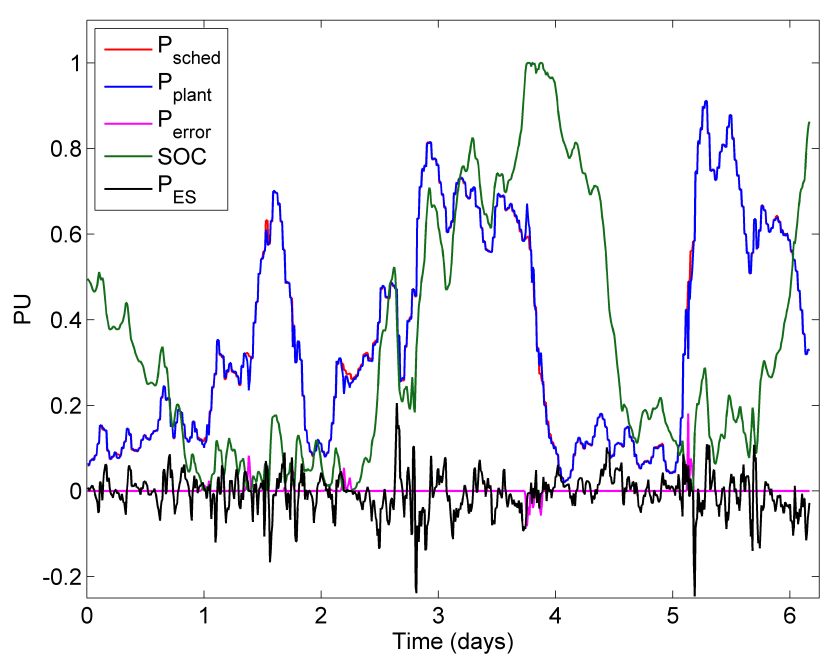

(c)

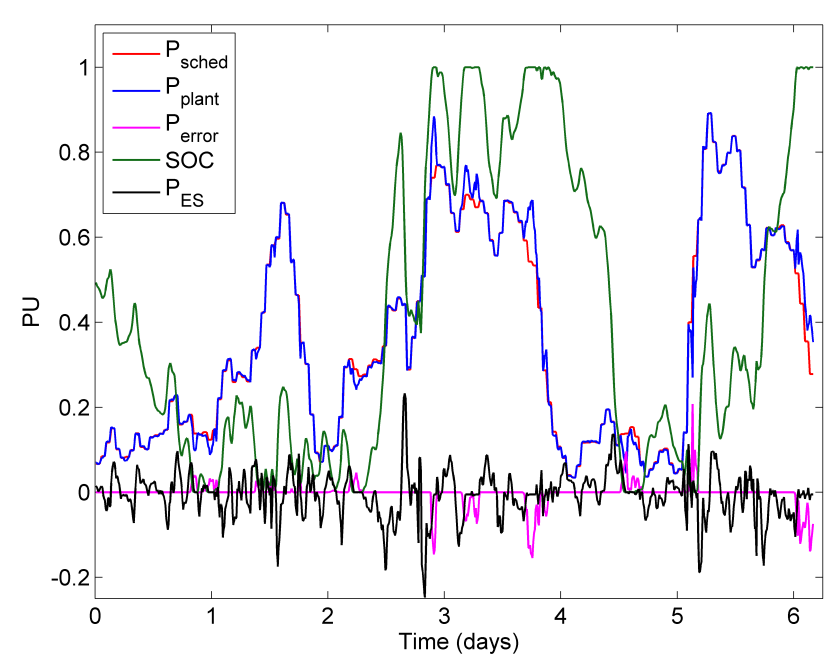

(b)

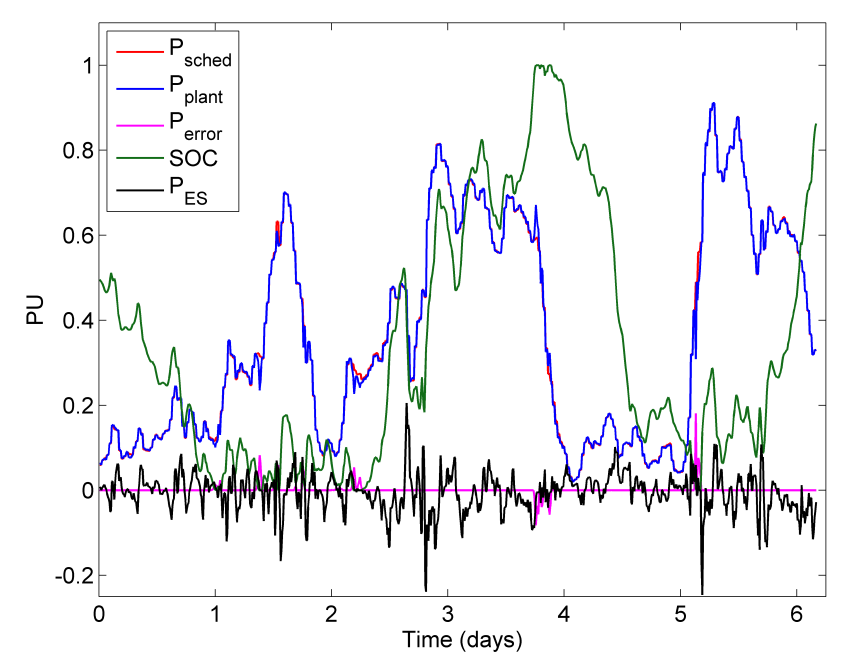

(d)

The addition of MPC, as opposed to simple reactive control, also makes a significant difference but is better observed quantitatively in Table 1. 
Table 1. Simulation results over approximately one week (888 10-min samples). Percent change from the base case, Case 1, is shown in parenthesis. MAE: mean absolute error.

\begin{tabular}{cccccc}
\hline Case & $\mathbf{1}$ & $\mathbf{2}$ & $\mathbf{3}$ & $\mathbf{4}$ & $\mathbf{5}$ \\
\hline Scheduling interval & $60 \mathrm{~min}$ & $60 \mathrm{~min}$ & $60 \mathrm{~min}$ & $30 \mathrm{~min}$ & $30 \mathrm{~min}$ \\
Energy storage & Inactive & Active & Active & Active & Active \\
Predictive control & NA & Off & On & Off & On \\
$P_{\text {err }}$ MAE & 0.0454 & $0.0086(-81)$ & $0.0088(-81)$ & $0.0021(-95)$ & $0.0022(-95)$ \\
$J_{\text {Q,err }}(\$)$ & 8211 & $1670(-80)$ & $1546(-81)$ & $346(-96)$ & $275(-97)$ \\
Following reserve (PU) & 0.2338 & $0.1467(-37)$ & $0.1039(-56)$ & $0.0937(-60)$ & $0.0573(-75)$ \\
Imbalance reserve (PU) & 0.3221 & $0.2375(-26)$ & $0.2290(-29)$ & $0.1335(-59)$ & $0.1272(-61)$ \\
\hline
\end{tabular}

Table 1 shows the results corresponding to the five cases. Four different metrics were examined: mean absolute error (MAE) for the $P_{\text {err }}$ time series, the error cost, and the following and imbalance reserve requirements. The error cost is defined below. In this analysis, $N$ equals 888 , which is the number of 10-min time steps used from the week of 26 March 2012:

$$
J_{\mathrm{Q}, \mathrm{err}}=\sum_{i}^{N} q_{P_{\mathrm{err}}} P_{\mathrm{err}}(i)^{2}
$$

Following reserves are calculated by taking the difference between hourly average power and $10 \mathrm{~min}$ power, whereas imbalance reserves are the difference between the scheduled hourly power and the hourly average power actually produced. The reserve metrics in the table represent the sum of the incremental (inc) and decremental (dec) reserves, which gives the amount of additional generation that must be available to either increase (inc) or decrease (dec) to balance wind fluctuations [20,21].

Case 1 in Table 1 presents the metrics for the base case, with no energy storage sources active. Case 2 presents the results with energy storage enabled and with predictive control off. In the cases without predictive control, the prediction horizon is effectively set to zero, such that the control command only corrects the error at the current time step (with no optimization of future time steps). Comparing the metrics in these two cases, it is clear that the addition of energy storage can significantly reduce error and improve reserve requirements (e.g., reducing the following reserve by $37 \%$ ).

Case 3 in Table 1 presents the results with predictive control enabled. Based on empirical analysis, a prediction horizon $\left(H_{\mathrm{p}}\right)$ of $2 \mathrm{~h}$ was chosen for the predictive control cases. Comparing Case 3 with Case 2, it is clear that, while predictive control does not have a major impact on the error-merely a very slight improvement in $J_{\mathrm{Q}, \text { err }}$ - there is an improvement in reserves. This is because $J_{\mathrm{Q}, \text { err }}$ is a weighted sum of all quadratic errors and is thus not generally dominated by any single sample. The reserve calculation on the other hand is largely determined by outlier events. For example, the single largest deviation within the hour with respect to the average over the entire year dictates the value of following reserve. Therefore we can see that the primary value of the predictive element is an improvement in the boundary (i.e., outlier) cases. The strength of the predictive controller becomes more apparent when the number of optimization objectives and constraints increases (as presented in the following sections). Case 4 is provided for completeness to show the results for a scheduling interval of 30 min with predictive control off (figure not shown). Case 5 presents the results for a scheduling interval of 30 min with 
predictive control on. Finally, a comparison of Cases 3 and 5 shows the benefit of the scheduling horizon reduction, for which there is approximately a four-fold reduction in the $P_{\text {err }}$ MAE. Therefore, we can see that the scheduling horizon is a very significant factor. The trend in the utility industry in the US is to shorten scheduling times. This effectively shifts the burden of variability from reserve generation to scheduled generation, so this may be a larger factor in reducing reserve requirements for wind farms than energy storage technology in the future.

\subsection{Minimizing $\Delta P_{\text {plant }}$}

In order to examine the predictive controller's ability to minimize wind ramps, the cost of plant ramps, $q_{\Delta P_{\text {plant }}}$, was set to a non-zero value. This enabled the controller to minimize ramp events by considering the cost of those events in the control action. A ramp was defined as a 0.20 PU change in plant output over $1 \mathrm{~h}$. This is based on the BPA definition of persistent deviation found in [19]. Figure 5(a),5(b) can be compared to see the difference between error reduction and ramp reduction; Cases 3 and 6, respectively. The black trace in each figure shows the $\Delta P_{\text {plant }}$ time series. It is clear that in the error reduction case (Figure 5(a)), $\Delta P_{\text {plant }}$ is uncontrolled, while in the ramp reduction case (Figure 5(b)), $\Delta P_{\text {plant }}$ is minimized (at the expense of $P_{\text {err }}$ ). The $P_{\text {plant }}$ time series in Figure 5(b) has much slower gradual changes over time compared with Figure 5(a).

Figure 5. Simulation results for: (a) Case 3 with $\Delta P_{\text {plant }}$ plotted; in this case, only $P_{\text {err }}$ is weighted; minimizing ramps is not considered in the control action; and (b) Case 6; in this case, only $\Delta P_{\text {plant }}$ is weighted; ramps are minimized in this case at the expense of $P_{\text {err }}$, as is evident in the plateau shape of $P_{\text {plant }}$.

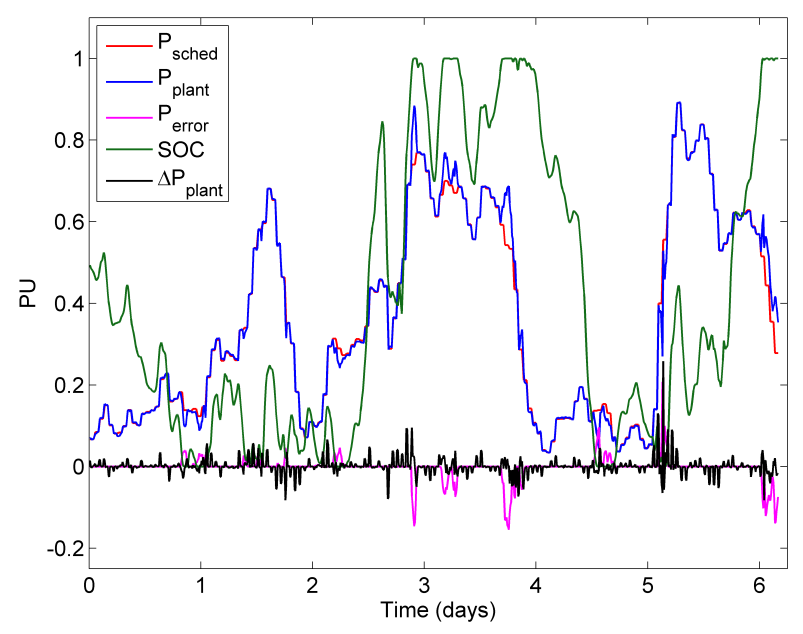

(a)

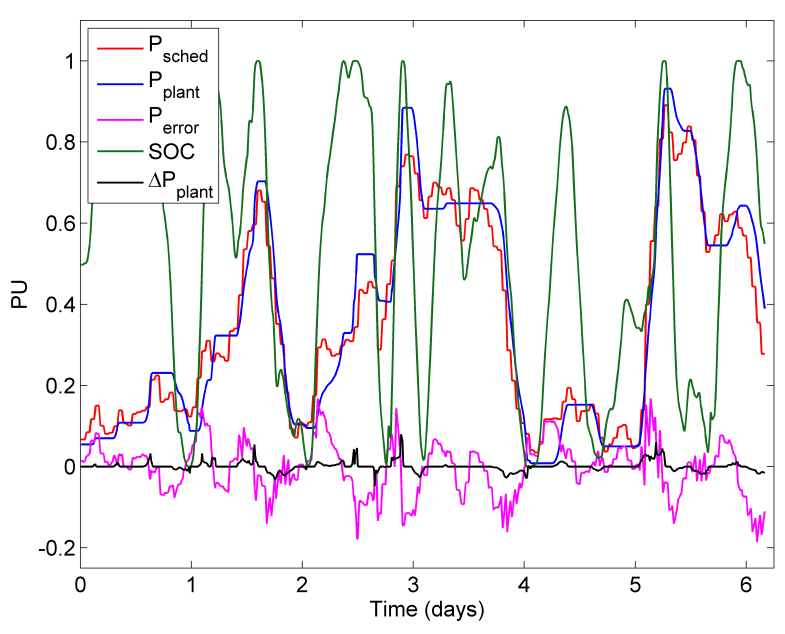

(b)

Quantitative results for ramp reduction are shown in Table 2. 
Table 2. Summary of error reduction, ramp reduction, and combined optimization cases over approximately one week (888 10-min samples). Percent change from the base case, Case 1, is given in parenthesis. Comparing Cases 7 and 8 with Case 3, it is shown that ramp events can be reduced with only a minimal impact on scheduling error. Compared with the base case, Case 1, it is shown that the scheduling error and the number of ramp events can be simultaneously improved.

\begin{tabular}{cccccc}
\hline Case & $\mathbf{1}$ & $\mathbf{3}$ & $\mathbf{6}$ & $\mathbf{7}$ & $\mathbf{8}$ \\
\hline Objective & Base case & Error reduction & Ramp reduction & \multicolumn{2}{c}{ Combined optimization } \\
Energy storage & Inactive & Active & Active & Active & Active \\
Error cost $\left(q_{P_{\text {err }}}\right)$ & 2503 & 2503 & 0 & 2503 & 2503 \\
Ramp cost $\left(q_{\Delta P_{\text {plant }}}\right)$ & 0 & 0 & 600 & 600 & 6000 \\
$P_{\text {err }}$ MAE & 0.0454 & $0.0088(-81)$ & $0.0515(+13)$ & $0.0101(-78)$ & $0.0141(-69)$ \\
$J_{\text {Q,err }} \quad(\$)$ & 8211 & $1546(-81)$ & 0 & $1563(-81)$ & $1859(-77)$ \\
$J_{\text {Q,ramp }}(\$)$ & 0 & 0 & 68 & 183 & 1133 \\
$J_{\text {Q,err }}+J_{\text {Q,ramp }}(\$)$ & 8211 & $1546(-81)$ & $68(-99)$ & $1746(-79)$ & $2992(-64)$ \\
Ramps up & 53 & $45(-15)$ & $14(-74)$ & $32(-40)$ & $16(-70)$ \\
Ramps down & 55 & $39(-29)$ & $1(-98)$ & $30(-45)$ & $12(-78)$ \\
Total ramps & 108 & $84(-22)$ & $15(-86)$ & $62(-43)$ & $28(-74)$ \\
\hline
\end{tabular}

\subsection{Minimizing $P_{\text {err }}$ and $\Delta P_{\text {plant }}$ (Combined)}

The strength of the MPC approach is best demonstrated when multiple objectives and constraints are simultaneously considered: minimizing error while also minimizing ramps. This scenario is significant because it shows that the addition of energy storage with proper control can reduce both error and ramping, which lessens the need for balancing from reserves. Figure 6(a),6(b) demonstrates two different combined optimization cases (Cases 7 and 8) where both error and wind ramps were simultaneously minimized by the controller. In Case 7 , the ramp cost is $600 \$ / \mathrm{PU}^{2}$ and in Case 8 the ramp cost is $6000 \$ / \mathrm{PU}^{2}$. The ramp costs for these cases were selected by sweeping the cost over a wide range and examining the resulting output parameters to experimentally determine appropriate values for presentation.

Similar to Table 1, various metrics are presented in order to quantify the performance of the controller. In addition to MAE of $P_{\text {err }}$, the table shows a breakdown of the cumulative error $\left(J_{\mathrm{Q}, \text { err }}\right)$ and cumulative ramp $\left(J_{\mathrm{Q}, \text { ramp }}\right)$ costs, as well as the total cost for each case. Equation (18) is the cumulative error cost, and the cumulative ramp cost is defined in Equation (19):

$$
J_{\text {Q,ramp }}=\sum_{i}^{N} q_{\Delta P_{\text {plant }}} \Delta P_{\text {plant }}(i)^{2}
$$

The last three rows of the table present a count of the number of up, down, and total ramps, where a ramp is defined as a $0.20 \mathrm{PU}$ change (or greater) over $1 \mathrm{~h}$, and the ramp count is determined through a sliding window over the $P_{\text {plant }}$ time series.

In Table 2, Cases 1 and 3 are presented again as before, and Cases 6, 7 and 8, which address ramping, are presented as well. It is clear that with only a minimal increase in the overall error (from an MAE 
of 0.0088 to an MAE of 0.0141 ), the number of ramp events can be significantly decreased from 84 in Case 3 (error reduction only) to 28 in Case 8 (combined error and ramp reduction).

Figure 6. Simulation results for: (a) Case 7 with combined error reduction and ramp reduction, with a ramp cost of $600 \$ / \mathrm{PU}^{2}$; and (b) Case 8 with combined error reduction and ramp reduction, with a ramp cost of $6000 \$ / \mathrm{PU}^{2}$. As shown in Table 2 , the ramp events are greatly reduced with a minimal increase in $P_{\text {err }}$.

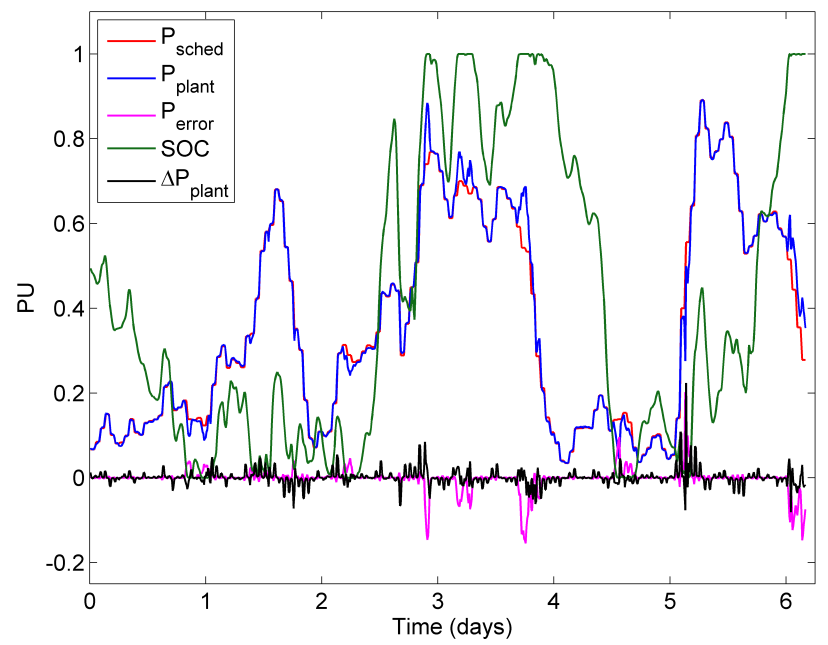

(a)

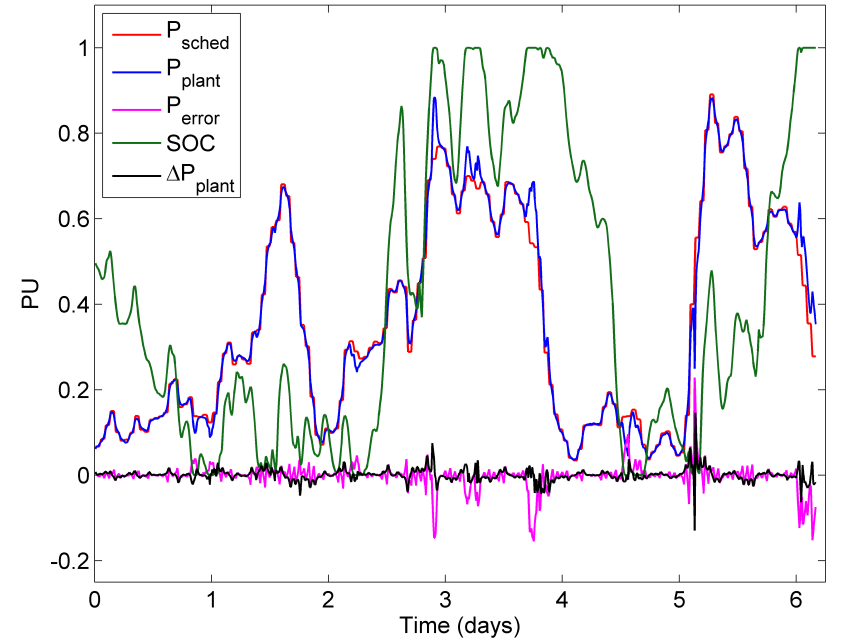

(b)

\section{Conclusions}

This paper presents an analysis of energy storage and MPC for improving the dispatchability of a large-scale wind farm. It has been shown that large-scale energy storage can be utilized to provide on-site balancing reserves for a wind farm. Energy storage with predictive control can reduce the scheduling error by approximately $80 \%$ and decrease the following reserve and the imbalance reserve by approximately $35 \%$ and $25 \%$, respectively (compared with a base case of no on-site energy storage).

One of the significant advantages of MPC is the ability to simultaneously consider several objectives in the context of system constraints. This has also been demonstrated by a reduction in scheduling error of approximately $70 \%$ and a simultaneous reduction in ramp events of approximately $75 \%$, when both aspects are weighted in the cost function. Increasing the weight on ramping will reduce ramping but cause more scheduling error. Increasing the weight on scheduling error will reduce scheduling error but cause more ramping.

It is also demonstrated that the scheduling horizon has a very significant impact on the scheduling error and the reserve requirements. Decreasing the scheduling horizon from $60 \mathrm{~min}$ to $30 \mathrm{~min}$ improves the scheduling error by an approximately additional $15 \%$ and improves the reserve requirements by an approximately additional $20 \%$ or more. Since the scheduling horizon is decided upon by the balancing area authority, it is not a degree of freedom for the energy storage system designer, but it is an important constraint that has a large impact on the system performance. Shorter scheduling horizons shift the burden of variability away from reserves to scheduled generation, thus possibly reducing the need for on-site energy storage for wind farms. 
Overall, the research shows that MPC is very well suited for energy storage control of providing reserves for variable, renewable power generation.

\section{Acknowledgments}

This work was supported in part by the BPA, Central Lincoln People's Utility District (PUD), Pacific Power, Portland General Electric (PGE), and Oregon Built Environment and Sustainable Technologies (BEST). Aspects of this work were also supported by the National Science Foundation under Grant No. 0846533.

\section{Author Contributions}

Simulation and analysis was largely conducted by graduate students Douglas Halamay and Michael Antonishen. Graduate students Kelcey Lajoie and Arne Bostrom provided writing support. Research formulation and direction was provided by Associate Professor and graduate student advisor Ted K.A. Brekken.

\section{Appendix}

A sample of the forecast and actual wind data over a 24-h period is presented in Figure A1 for reference. While new forecast data over the 2-h prediction horizon is available at every 10 -min time step, only the forecasts for every sixth time step (each hour) are presented in the figure to improve clarity (in alternating green and red colors). Since each forecast is composed of 12 data points $(2 \mathrm{~h}$ ), the forecast data overlap. With a new forecast at each time step, the predictive controller is able to refine its control actions to efficiently utilize the energy storage source.

Figure A1. Example of actual $\left(P_{\text {farm }}\right) v s$. forecasted wind data over a single day. Note that a new forecast is made at each 10-min time step. For clarity, however, only the forecast data from every sixth time step (each hour) is presented (in alternating green and red colors to show the overlapping nature of the forecasts).

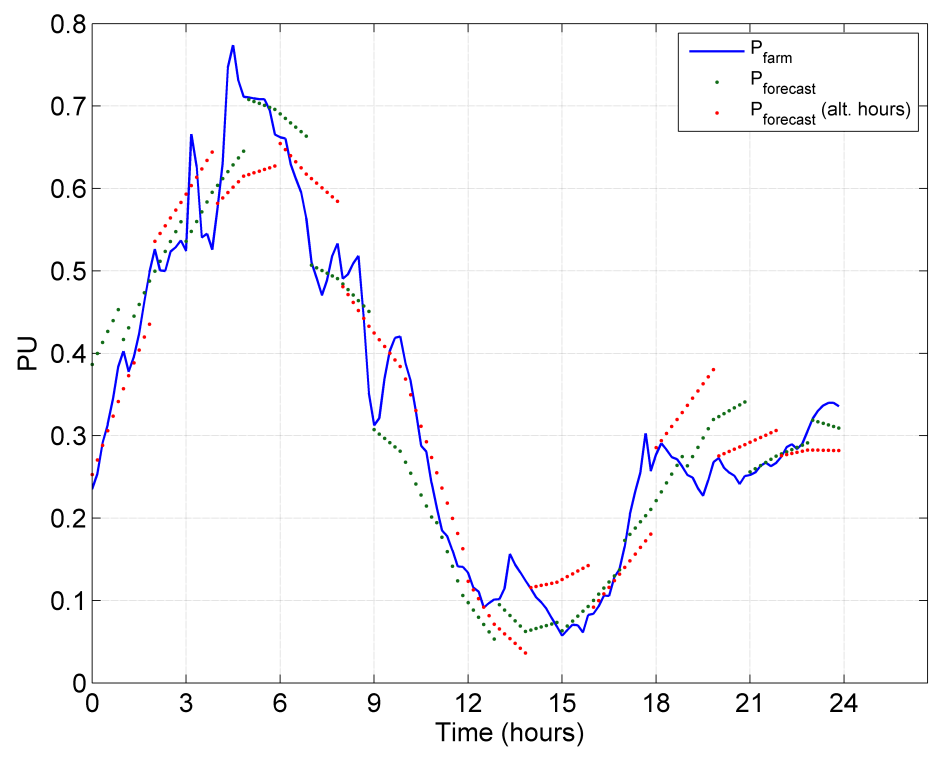




\section{Conflicts of Interest}

The authors declare no conflict of interest.

\section{References}

1. Wind Generation Capacity, 2012. Available online: http://transmission.bpa.gov/business/ operations/Wind/WIND_InstalledCapacity_PLOT.pdf (accessed on 1 June 2013).

2. Connecting Variable Generating Resources to the Federal Columbia River Transmission System (FCRTS); Bonneville Power Administration (BPA): Portland, OR, USA, 2010.

3. Dragoon, K.; Charles, G. Historical Energy Production; Northwest Power and Conservation Council (NPCC): Portland, OR, USA, 2012.

4. Current and Proposed Wind Project Interconnections to BPA Transmission Facilities; Bonneville Power Administration (BPA): Portland, OR, USA, 2011.

5. Han, H.; Brekken, T.K.A.; von Jouanne, A.; Bistrika, A.; Yokochi, A. In-lab research grid for optimization and control of wind and energy storage systems. In Proceedings of the 2010 49th IEEE Conference on Decision and Control (CDC), Atlanta, GA, USA, 15-17 December 2010; pp. 200-205.

6. Khatamianfar, A.; Khalid, M.; Savkin, A.; Agelidis, V. Improving wind farm dispatch in the Australian electricity market with battery energy storage using model predictive control. IEEE Trans. Sustain. Energy 2013, 4, 745-755.

7. Perez, E.; Beltran, H.; Aparicio, N.; Rodriguez, P. Predictive power control for PV plants with energy storage. IEEE Trans. Sustain. Energy 2013, 4, 482-490.

8. Qi, W.; Liu, J.; Christofides, P. Supervisory predictive control for long-term scheduling of an integrated wind/solar energy generation and water desalination system. IEEE Trans. Control Syst. Technol. 2012, 20, 504-512.

9. Thatte, A.; Zhang, F.; Xie, L. Coordination of wind farms and flywheels for energy balancing and frequency regulation. In Proceedings of the 2011 IEEE Power and Energy Society General Meeting, San Diego, CA, USA, 24-29 July 2011; pp. 1-7.

10. Teleke, S.; Baran, M.E.; Bhattacharya, S.; Huang, A.Q. Optimal control of battery energy storage for wind farm dispatching. IEEE Trans. Energy Convers. 2010, 25, 787-794.

11. Baone, C.; DeMarco, C. Saturation-bandwidth tradeoffs in grid frequency regulation for wind generation with energy storage. In Proceedings of the 2011 IEEE PES Innovative Smart Grid Technologies (ISGT), Hilton Anaheim, CA, USA, 17-19 January 2011; pp. 1-7.

12. Mendis, N.; Muttaqi, K.; Sayeef, S.; Perera, S. Control coordination of a wind turbine generator and a battery storage unit in a remote area power aupply system. In Proceedings of the 2010 IEEE Power and Energy Society General Meeting, Minneapolis, MN, USA, 25-29 July 2010; pp. $1-7$.

13. Banham-Hall, D.D.; Taylor, G.A.; Smith, C.A.; Irving, M.R. Frequency control using vanadium redox flow batteries on wind farms. In Proceedings of the 2011 IEEE Power and Energy Society General Meeting, San Diego, CA, USA, 24-29 July 2011; pp. 1-8. 
14. Fazeli, M.; Asher, G.; Klumpner, C.; Yao, L.; Bazargan, M.; Parashar, M. Novel control scheme for wind generation with energy storage supplying a given demand power. In Proceedings of the 2010 14th Power Electronics and Motion Control Conference (EPE/PEMC), Ohrid, Republic of Macedonia, 6-8 September 2010; pp. S14-15-S14-21.

15. Rossiter, J. Model-based Predictive Control: A Practical Approach; CRC Press: Boca Raton, FL, USA, 2003.

16. Antonishen, M.; Han, H.; Brekken, T.; von Jouanne, A.; Yokochi, A.; Halamay, D.; Song, J.; Naviaux, D.; Davidson, J.; Bistrika, A. A methodology to enable wind farm participation in automatic generation control using energy storage devices. In Proceedings of the 2012 IEEE Power and Energy Society General Meeting, San Diego, CA, USA, 22-26 July 2012; pp. 1-7.

17. Brekken, T.K.A.; Yokochi, A.; von Jouanne, A.; Yen, Z.; Hapke, H.; Halamay, D. Optimal energy storage sizing and control for wind power applications. IEEE Trans. Sustain. Energy 2011, 2, 69-77.

18. BPA Wind Generation in the Last 7 Days, 2012. Available online: http://transmission.bpa.gov/ Business/Operations/Wind/baltwg.aspx (accessed on 1 June 2013).

19. 2012 BPA Final Rate Proposal, Generation Inputs Study; Bonneville Power Administration (BPA): Portland, OR, USA, 2011.

20. Reserve Capacity Forecast for Wind Generation within-hour Balancing Service; Bonneville Power Administration (BPA): Portland, OR, USA, 2008.

21. Balancing and Frequency Control; North American Electric Reliability Corporation (NERC): Atlanta, GA, USA, 2011.

(c) 2014 by the authors; licensee MDPI, Basel, Switzerland. This article is an open access article distributed under the terms and conditions of the Creative Commons Attribution license (http://creativecommons.org/licenses/by/3.0/). 